\title{
INITIAL VALUES OF SOLUTIONS OF THE NAVIER-STOKES EQUATIONS
}

\author{
CALIXTO P. CALDERÓN
}

(Communicated by J. Marshall Ash)

\begin{abstract}
This paper considers weak solutions to the Navier-Stokes equations in the sense considered in previous papers of the author and of Fabes, Jones, and Rivière. Results concerning pointwise a.e. convergence of the solutions to the initial values are established. The initial values that are considered here are divergence free vector functions belonging to $L^{p}\left(R^{n}\right)$, for $p$ greater than or equal to the dimension $n$.
\end{abstract}

In this paper we consider weak solutions to the Navier-Stokes equations in the sense considered in the papers [2, 3, 5]. In fact, the problem consists in establishing results concerning pointwise convergence a.e. of the weak solutions to the initial values. The problem was proposed to me by Rivière who, together with Fabes and Jones, wrote a landmark paper on the existence of weak solutions for the Navier-Stokes equations with initial data in $L^{p}$, see reference [5]. I will invoke that paper, [5], as well as my papers $[2,3]$ concerning the matters and results to be discussed here.

We shall be concerned with solutions to the initial value problem for the N-S equations in the infinite cylinder $S_{T}=R^{n} \times[0, T)$ for the initial values

$$
f(x)=\left(f_{1}(x), f_{2}(x), \ldots, f_{n}(x)\right),
$$

where $f(x)$ is a divergence free vector function belonging to $L^{p}$ of $R^{n}$. More precisely, the initial data should satisfy $\int_{R^{n}}|f|^{p} d x$ finite, $p \geq n$, and

$$
\operatorname{div} f=0 \text { in the distribution sense. }
$$

Weak solutions $u(x, t)$, for the N-S equations for those initial values have been found in various functional spaces, at least for a small period of time, namely, $0<t<T$. For these results we refer the reader to papers $[5,2,3]$. The norms that will be useful here have already been introduced in $[2,3]$. They are

$$
\left\|u^{*}\right\|_{p}(T)=\left(\int_{R^{n}} \sup _{0<t<T}|u|^{p} d x\right)^{1 / p}, \quad p \geq n,
$$

where $u^{*}(x)=\sup _{0<t<T}|u(x, t)|$.

Received by the editors April 18, 1990 and, in revised form, June 28, 1991.

1991 Mathematics Subject Classification. Primary 35Q10, 35K55, 35K15. 
Also, we consider the space of divergence free, weak vector solutions $u(x, t)$ to the N-S equations in the cylinder $s_{T}$ for divergence free initial data in $L^{p}$, that is, functions satisfying

$$
\operatorname{div} u=0 \quad \text { in the distribution sense }
$$

and

$$
D_{t} u_{i}-\sum_{j=1}^{j=n} D_{j j} u_{i}+\sum_{j=1}^{j=n} u_{j} D_{j} u_{i}+D_{i} p=0, \quad u(x, 0)=f(x) .
$$

Hence, $D_{j}$ and $D_{t}$ denote, respectively, the distributional derivatives with respect to $s_{j}$ and $t$; likewise $D_{i j}$ will denote the second derivative with respect to the spatial coordinates $x_{i}$ and $x_{j} ; p$ denotes the pressure. If $L(u)$ denotes the heat operator applied to $u$ and $\operatorname{grad} u$ denotes the square matrix $D_{j} u_{i}$, then equation $(0.3)$ can be written as

$$
L(u)+(\operatorname{grad} u)(u)+\operatorname{grad} p=0 .
$$

A vector function $u(x, t)$ is said to be a weak solution of the N-S equations, with initial values $f$, $\operatorname{div} f=0$, if for any $C^{\infty}$, rapidly decreasing vector function $v(x, t)$ defined on $R^{n+1}$, such that $\operatorname{div} v=0$ there, and $v=0$ if $t>T$, we have

$$
\left[\int_{0}^{T}\left(\|u\|_{p}\right)^{q} d t\right]^{1 / q} \text { is finite }
$$

(where the norm inside the integral is taken over $R^{n}$ on the spatial coordinates, $p$ and $q$ greater than or equal to 2 ) and

$$
\begin{gathered}
\int_{0}^{T} \int_{R^{n}}\left\langle u, L^{*}(v)+(\operatorname{grad} v)(u)\right\rangle d x d t \\
=-\int_{R^{n}}\langle f(x), v(x, 0)\rangle d x .
\end{gathered}
$$

$L^{*}$ stands for the adjoint heat operator. In addition, $u$ has to be divergence free.

\section{Main Results}

The main result is contained in

Theorem I. Let $u(x, t)$ be a weak solution to equation (0.3) for the initial values $f$, divergence free and belonging to $L^{n}$ of $R^{n}$. Then there exists a time $T$ depending on $f$ and such that

(i) $\left\|u^{*}\right\|_{n}(T)$ is finite;

(ii) the function $u(x, t)$ converges a.e. to $f(x)$ as $t$ goes to 0 .

The existence of a solution $u$ is a consequence of Theorem B in [2]. If the initial data $f$ belong to $L^{p}, p>n$, a similar result holds but with the $L^{p}$ norm replacing the $L^{n}$ norm in (i) above. In the case $p>n$, the size of $T$ depends only on the $L^{p}$ norm of $f$.

2. FUNDAMENTAL SOLUTIONS, THE BILINEAR FORM, AND FIXED POINTS

Fabes, Jones, and Rivière extended to dimension $n$ a formula found for the case $n=3$ by Oseen. The Oseen-Fabes-Jones-Rivière formula provides a 
divergence free matrix fundamental solution $E_{i j}(x, t)$ for the $n$-dimensional heat equation. The definition of the fundamental divergence free fundamental solution $E$ should be consulted in references [2, 3, 5]. I will list here the main properties of $E$ :

1. $E$ is symmetric and divergence free; namely, $E_{i j}=E_{j i}, \sum_{j=1}^{j=n} D_{j} E_{i j}(x, t)$ $=0$, if $t>0$, we take ordinary derivatives.

2. The bilinear form $B(u, u)$, defined as

$$
\int_{0}^{t} \int_{R^{n}}\langle(\operatorname{grad} E(x-y, t-s))(u(y, s)), u(y, s)\rangle d y d s,
$$

allows us to write in a condensed form the fundamental integral equation of this theory, namely,

$$
u+B(u, u)=F(x, t) .
$$

Here, $F(x, t)$ stands for the convolution in the spatial variables of the initial data with the classical fundamental solution $W(x, t)$ of the heat equation, namely,

$$
F(x, t)=\int_{R^{n}} W(x-y, t) f(y) d y .
$$

A very important result in [5] asserts that $u$ is a weak solution of equation (0.3) for the initial data $f$, divergence free and in $L^{p}$ of $R^{n}, p>1$, if and only if it is a solution of the integral equation (2.1).

3 . The study of the integral equation (2.1) leads in a natural way to considering perturbed linear operators and bilinear operators of the form $T(u, v)=$ $B(u, v)+1(u)+F(x, t)$. Here, $B$ is the bilinear operator defined in paragraph $2,1(u)$ is a linear operator, and $F$ has already been defined above. The domains of these operators will be the Banach spaces of measurable vector functions for which the norm $(0.2)$ is finite for some $p$. We shall be mainly interested in the class of norms for which the following natural estimate holds:

$$
\|T(u, v)\|<C_{1}\|u\|\|v\|+C_{2}\|u\|+\|F\| .
$$

The following lemma has been invoked in [3] and a proof of it can be found in [2].

Lemma 1. The quadratic operator $T(u, u)$ maps the ball $\left\{u ;\|u\|<s_{1}\right\}$ into itself, provided that the following is satisfied:

$$
\left[1-C_{2}\right]^{2}>4 C_{1}\|F\|, \quad C_{1}>0,0<C_{2}<1 .
$$

The operator $T$ and the coefficients $C_{1}, C_{2}$, and $F$ are as defined above and satisfying (2.3). The number $s_{1}$ is the smaller root of the equation

$$
C_{1} s^{2}+\left(C_{2}-1\right) s+\|F\|=0 .
$$

If $2 s_{1} C_{1}+C_{2}<1$, then the operator $T$ is a contraction mapping in the ball of radius $s_{1}$. The estimate for $s_{1}$,

$$
s_{1}<\|F\|\left[\left(1-C_{2}\right)^{2}-4 C_{1}\|F\|\right]^{-1 / 2}=A,
$$

gives a very simple condition for $T$ being a contraction mapping, namely,

$$
2 A C_{1}+C_{2}<1 \text {. }
$$




\section{ESTIMATES FOR THE BILINEAR FORM $B(u, v)$}

The following domination is standard and can be found in [5]:

$$
|\operatorname{grad} E|<C|x|^{a-n} t^{-(1+a) / 2}, \quad t>0,0<a<1 .
$$

Calling $u^{*}(x, T)=\sup _{0<t<T}|u(x, t)|$, one can readily verify

$$
|B(u, v)|<C T^{1-(1+a) / 2}\left(|x|^{a-n}\right) *\left(u^{*}(T) v^{*}(T)\right) .
$$

In the above formula, * denotes convolution in the space coordinates. In what follows we shall use a bilinear form of Sobolev's inequality, namely,

$$
\left\||x|^{a-n} *(f g)\right\|_{r}<C\|f\|_{p}\|g\|_{q}, \quad \frac{1}{r}=\frac{1}{p}+\frac{1}{q}-\frac{a}{n},
$$

where $C$ stands for a constant and the norms are the usual Lebesgue norms in $R^{n}$ (the spatial coordinates).

The above inequality can be applied to the transformation

$$
T(u, v)=B(u, v)+F(x, t),
$$

where $B(u, v)$ stands for the bilinear form defined in II above, $F$ denotes as before the convolution of the initial data $f$ with the classical fundamental solution of the heat equation $W$.

The estimates (3.1), (3.2), (3.3), (3.4), Lemma 1, and the maximal theorem of Hardy-Littlewood applied to $W * f=F$ prove

Theorem II. The integral equation (2.1) has a unique solution $u$ on $S_{T}$ for the divergence free initial values $f$ in $L^{p}, p=n / a$. The size of $T$ depends on the norm of the initial values. Moreover, we have $\left\|u^{*}\right\|_{p}(T)<A$ where $p=n / a$, $0<a<1$, and $A$ is the constant defined in (2.4). The estimate (3.2) and the fact that $F=W * f$ give the pointwise convergence of $u(x, t)$ to $f$ as tends to zero. On account of the Fabes-Jones-Riviere representation theorem for weak solutions of the N-S equations, we obtain the thesis of Theorem I for the case $f$ in $L^{p}, p>n$.

\section{END OF THE PROOF OF THEOREM I}

The proof for the case of initial data in $L^{n}$ proceeds with the idea of splitting the initial data and the equations. The initial values are going to be split into two vector functions preserving the divergence free structure of the original data. One part, with large $L^{n}$ norm, is going to be embedded in $L^{p}, p=n / a$, $0<a<1$. This part will be handled with Theorem II above. The other part is going to remain in $L^{n}$ and have small norm there. This part will be a solution of a perturbed N-S equation, but more conveniently, it will be a solution of a modified form of equation (2.1).

1. Splitting the initial data. Let us choose a large $s>0$ and partition $f$ as $f_{j}=f_{j}^{(1)}+f_{j}^{(2)}$. The component $f^{(1)} j$ equals $f_{j}$ if $\left|f_{j}\right|<s$ and zero otherwise.

The function $g$ is defined by the equations

$$
g_{i}=\lim \sum_{j} \int E_{i j}(x-y, t) f_{j}^{(1)}(y) d y .
$$

In the above expression the limit is understood in $L^{p}$ for $t$ tending to zero. A similar transformation defines $h_{j}$ in terms of $f^{(2)} j$. The singular integral 
transformation in (4.1) has been studied in [2] and constitutes a bounded linear map from $L^{p}$ into itself for finite $p>1$. For details the reader should consult [2, Lemma II.1].

From the construction and the properties of $E$, one can verify at once that

(i) $f=h+g$ a.e., $f$ and $g$ are divergence free.

(ii) If $s$ is selected large then the $L^{n}$ norm of $h$ can be made arbitrarily small.

(iii) $g$ is the function that is embedded in $L^{p}, p=n / a$.

2. The second step is to solve the equation $u+B(u, u)=W * g$. We use Theorem II for solving this equation and find small $T$, depending on the $L^{p}$ norm of $g$.

3. The third step is to solve for $v$ the equation

$$
v+B(v, u)+B(u, v)+B(v, v)=W * h .
$$

Here, the function $u$ has been determined from step 2, and, in order to find $v$ we have to apply Lemma 1 in $\S 2$ and the inequalities (3.2) and (3.3) in the following way:

$$
\left\|B(v, u)^{*}\right\|_{n}\left(T^{\prime}\right)<C T^{\prime 1-(1+a) / 2}\left\|u^{*}\right\|_{p}\left(T^{\prime}\right)\left\|v^{*}\right\|_{n}\left(T^{\prime}\right) .
$$

A similar inequality holds for $B(u, v)$. It should be noticed that we may have to take $T^{\prime}$ smaller than the $T$ obtained for $u$.

Finally, as a consequence of Lemma III.1 in [2], we obtain for $B(v, v)$ a stronger inequality, namely,

$$
\left\|B(v, v)^{*}\right\|_{n}<C\left\|v^{*}\right\|_{n}\left\|v^{*}\right\|_{n} .
$$

In the above inequality the sup can be taken over all time $t>0$. More precisely,

$$
|B(v, v)(x, t)|<C\left(|x|^{1-n} *\left(v^{*}\right)^{2}(T)\right), \quad 0<t<T .
$$

The above inequality, with $C$ independent from $T$, is a consequence of the sharper one (see [2, Lemma III.1])

$$
\begin{gathered}
|B(v, v)(x, t)|<C \int_{R^{n}} K(x-y, t)|x-y|^{1-n}\left(v^{*}(T)\right)^{2} d y, \\
K(y, t)=\int_{0}^{t}|y|^{-2}\left(1+|y|^{-1} s^{1 / 2}\right)^{-1-n} d s
\end{gathered}
$$

valid for the range of values $0<t<T$. The estimates (4.2), (4.3), (4.5) which are also valid for the fixed point $v$ and the representation $W * h$, give the pointwise convergence a.e. as $t$ tends to zero. This concludes the proof of Theorem I.

\section{REFERENCES}

1. A. Benedek and R. Panzone, The spaces $L^{p}$ with mixed norm, Duke Math. J. 28 (1961), 301-324,

2. Calixto P. Calderón, Existence of weak solutions for the Navier-Stokes equations with initial data in $L^{p}$, Trans. Amer. Math. Soc. 318 (1990), 179-200

3. __ Addendum to the paper "Existence of weak solutions for the Navier-Stokes equations with initial data in $L^{p} "$, Trans. Amer. Math. Soc. 318 (1990), 201-207. 
4. E. B. Fabes and N. M. Rivière, Singular integrals with mixed homogeneity, Studia Math. 27 (1966), 19-38.

5. E. B. Fabes, B. F. Jones, and N. M. Rivière, The initial value problem for the Navier-Stokes equations with data in $L^{p}$, Arch. Rational Mech. Anal. 45 (1972), 222-240.

Department of Mathematics, Statistics and Computer Science, University of Illinois at Chicago, Chicago, Illinois 60680 